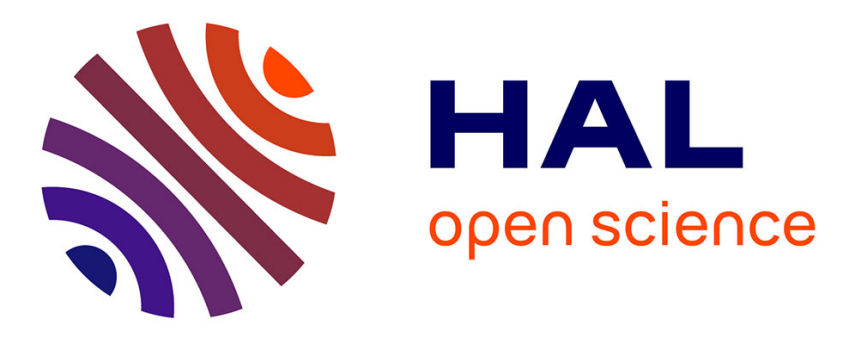

\title{
Pulmonary hemodynamics simulations before stage 2 single ventricle surgery: patient-specific parameter identification and clinical data assessment
}

Grégory Arbia, Chiara Corsini, Catriona Baker, Giancarlo Pennati, Tain-Yen Hsia, Irene Vignon-Clementel

\section{To cite this version:}

Grégory Arbia, Chiara Corsini, Catriona Baker, Giancarlo Pennati, Tain-Yen Hsia, et al.. Pulmonary hemodynamics simulations before stage 2 single ventricle surgery: patient-specific parameter identification and clinical data assessment. Cardiovascular Engineering and Technology, 2015, pp.18. 10.1007/s13239-015-0212-3 . hal-01063967

\section{HAL Id: hal-01063967 https://hal.inria.fr/hal-01063967}

Submitted on 15 Sep 2014

HAL is a multi-disciplinary open access archive for the deposit and dissemination of scientific research documents, whether they are published or not. The documents may come from teaching and research institutions in France or abroad, or from public or private research centers.
L'archive ouverte pluridisciplinaire HAL, est destinée au dépôt et à la diffusion de documents scientifiques de niveau recherche, publiés ou non, émanant des établissements d'enseignement et de recherche français ou étrangers, des laboratoires publics ou privés. 


\title{
Multiscale modeling of pulmonary hemodynamics in first stage single ventricle patients: integrating clinical data and simulations
}

\author{
Gregory Arbia - Chiara Corsini - Catriona \\ Baker - Giancarlo Pennati . Tain-Yen Hsia . \\ Irene E. Vignon-Clementel - for the Modeling of \\ Congenital Hearts Alliance (MOCHA) Investigators
}

the date of receipt and acceptance should be inserted later

\begin{abstract}
Purpose: Congenital heart defects involve pathologies in which the heart has only one functional pumping chamber. In these conditions, treatments consist of three procedures where stage 1 occurs just after birth and links the systemic and pulmonary circulations through an artificial shunt. Computational fluid dynamics is a tool to explore hemodynamics in patientspecific simulations, and understand specific physiopathologies. Representative hemodynamics models are built based on imaging data for example to explore several virtual surgical options. In this context, the specification of boundary conditions is necessary to compute pressure and flow in the entire domain. However, these boundary conditions are rarely the measured variables. Besides, to take into account the rest of the circulation outside of the three-dimensional modeled domain, a number of reduced order models exist.

Methods: In this work a method is presented to iteratively but automatically tune from clinically measured hemodynamics data reduced model parameters. Target pressures such as minimum, maximum or average over one or both lungs are considered, depending on the clinical measurement. A 5-parameter model representing more specifically the arterial and venous pulmonary circulation is proposed.

Results: The methodology is demonstrated with six stage 1 patient specific models: the clinical target data are well matched, except for one case where clinical data were incoherent. Inhomogeneous pressure, swirling blood flow patterns and very high wall shear stress 3D maps highlight similarities and differences among patients. Steady and pulsatile tuning results are similar.

Conclusions: The highly complex fluid dynamics of this stage is for the first time analyzed around the distal systemic-to-pulmonary shunt anastomosis and the connected pulmonary ar-

Gregory Arbia · Irene E. Vignon-Clementel

INRIA Paris-Rocquencourt, Le Chesnay Cedex, France and UPMC Univ Paris 6, Laboratoire Jacques-Louis Lions, Paris, France

Tel.: +331.39.63.51.18

Fax: +331.39.63.58.82

E-mail: irene.vignon-clementel@inria.fr

Chiara Corsini · Giancarlo Pennati

Laboratory of Biological Structure Mechanics, Department of Chemistry, Materials and Chemical Engineering 'Giulio Natta', Politecnico di Milano, Milan, Italy

Catriona Baker · Tain-Yen Hsia

Centre for Cardiovascular Imaging, UCL Institute of Cardiovascular Science, and Great Ormond Street Hospital for Children, NHS Foundation Trust, London, UK
\end{abstract}


teries. The distal vasculature of each outlet is better represented than previously. This work demonstrates how simulations can help to check the coherence of the clinical data or provide insights to clinicians that are otherwise difficult to measure, such as in the presence of kinks. A strategy for the case of incoherent clinical data is proposed to match the data. Finally, the choice of steady versus pulsatile tuning, limitations and possible extensions of this work are discussed.

Keywords Parameter estimation - Clinical hemodynamics data - Patient-specific CFD . Multiscale simulations · First stage single ventricle patients · Pulmonary circulation modelling

\section{Introduction}

Single ventricle defects are a spectrum of congenital heart disorders resulting in only one functional ventricle. Several stages of palliative surgery are required to connect the systemic and pulmonary circulations to the single ventricular power source. The first stage commonly utilises a shunt between the aorta, or its branches, and the pulmonary arteries. Accurate modeling of patient-specific physiology for clinical decision-making requires the integration of the patient's clinical data into numerical simulations [26].

Computational fluid dynamics studies of single-ventricle conditions $[5,25,27]$ have presented the crucial interest of specifying the relevant boundary conditions. More specifically in [10] authors investigated the effect of clinical parameters such as shunt, vasculature and heart rate on Norwood procedure. A common way to take into account the effect of the downstream domain is to couple 3D Navier-Stokes equations to electric-analog (also called zero-dimensional or reduced) models or one-dimensional models of the peripheral circulation $[16,24,25,26]$. This gives information about local fluid dynamics due to changes in anatomical features $[7,9]$. In [18] the authors presented computational hemodynamics simulations but in the systemic circulation of one specific Norwood case with aortic arch repair and Damus-Kay-Stansel anastomosis. The distribution of flows among the outlets was not directly controlled since a zero pressure gradient method was applied at the end of very long extensions to recover a physiological pressure level. The pressure results were compared with pressure measurements at three locations in the aorta.

A first idea consists of manually tuning parameters, which requires hemodynamics intuition [16] and has been experienced to be out of reach if there are many branches. Automatic parameter estimation methods have thus been developed. In [22], six parameters of an aortic model were estimated with a quasi-Newton method to achieve some pressure and flow waveform features inside the 3D model. In [8], the Windkessel parameters of 3D bifurcating aneurysm were estimated with an adjoint-based method to match systolic, diastolic and average pressure differences. In [4], wall displacement values were used to estimate the stiffness of the 3D fluid-solid interaction idealized aneurysm model and its outlet proximal resistance, based on a sequential estimation approach. In [15], a dozen of Windkessel parameters in a 3D coarctation model were estimated with a Kalman filter approach on a corresponding 0D surrogate model of the 3D-0D model, to match given flow and pressure waveforms inside the 3D domain.

In this paper we present a CFD analysis of the blood flow behavior in the distal anastomosis of the systemic-to-pulmonary shunt and the connected pulmonary arteries over several bifurcations, in six different patients. At the 3D outlets, it would not be relevant to apply pressure boundary conditions for the following reasons: 1) the measured flow distribution between the two different lungs cannot be easily matched, and 2) the pressure measurements were not performed at these locations in the pulmonary arteries. Sometimes, applying the same pressure at all outlets 
can even lead to unphysiological reverse average flow in some of the branches. Applying timevarying flow boundary conditions would also be difficult because the flow distribution between the different outlets is not necessarily constant, and would not guaranty to be coherent with the pressure measurements. Moreover, prescribing pressure or flow reduces the predictive potential such as in virtual surgery planning. We thus aim at coupling 3D Navier-Stokes equations to reduced models including resistances and capacitances. A previous work [23] introduced a method to iteratively tune reduced order parameters of the 3D model to match clinical inlet average pressure, inlet average flow and outlets' flow repartition. The method was illustrated on coupling of 3D steady Navier-Stokes equations to total outlet resistances of dozens of branches in Stage 2 (Glenn) patient-specific simulations. From these resistances, 3-parameter Windkessel models were generated to represent the downstream arterial tree, neglecting the venous circulation.

In this work we extend the method to iteratively tune reduced parameters for more general pressure clinical data localization, as clinical data are rarely given at the specific 3D boundaries. The methodology is demonstrated for steady and pulsatile cases, with clinical data of stage 1 patients. The highly complex fluid dynamics of this stage is analyzed. Moreover, the downstream reduced models of the pulmonary circulation is enriched to a 5-parameter model representing more specifically the arterial and venous circulations.

The article continues with the Methods section to present the different steps of the algorithm, from clinical data, automatic parameters tuning to the final reduced pulmonary model generation for each outlet. The Results section presents then the demonstration of this framework on six stage 1 single ventricle patients, reporting their specific 3D hemodynamics. A discussion follows on methodological, biomechanical and clinical aspects.

\section{Methods}

The Methods section presents the type of clinical data that define the patient-specific geometry and hemodynamics target values. The automatic reduced model parameters tuning to reach these target values is explained, followed by the 5-parameter reduced model description. In this work six patient-specific computational models were built to describe hemodynamics into the corresponding three-dimensional regions of interest, reflecting the patient state in coherence with the clinical measurements acquired before stage 2 surgery.

\subsection{Clinical cases}

Patients A, B and C were recruited at the University of Michigan, Ann Arbor, MI, USA; patients D and E at Medical University of South Carolina, Charleston, SC, USA; and patient F recruited at Great Ormond Street Hospital, London, UK. The study was approved by the institutional review board at each institution and consent was obtained from the subject's legal guardian.

Patient $\mathrm{A}$ had a diagnosis of pulmonary atresia with intact ventricular septum. The stage 1 surgery comprised a central shunt, from the ascending aorta (AoA) to the main pulmonary artery (MPA). This patient was 6 months old at time of cardiovascular magnetic resonance imaging (CMR), with body surface area (BSA) of $0.34 \mathrm{~m}^{2}$. Patients B, C, D and F had hypoplastic left heart syndrome. Their stage 1 operations were all $3.5 \mathrm{~mm}$ right modified Blalock-Taussig shunts (rmBT). These patients were 4, 5, 5 and 4 months old at time of CMR, with BSA of $0.28,0.34$, 0.30 and $0.27 \mathrm{~m}^{2}$ respectively. Patient $\mathrm{E}$ had tricuspid and pulmonary atresia, and underwent stage 1 surgery of a $4 \mathrm{~mm} \mathrm{rmBT}$, with additional left pulmonary artery patch to treat a left 
pulmonary artery stenosis. This patient was 3 months old at time of CMR, with BSA of 0.26 $\mathrm{m}^{2}$.

\subsection{Clinical measurements}

For patients A, B and C, CMR was performed immediately prior to the second surgery under the same general anesthetic, and cardiac catheterization was performed respectively 3 days, 1.5 months and 1 week prior to the second surgery. CMR and cardiac catheterization were performed on the same day, 1.5 months prior to the second surgery for patient $\mathrm{D}$; and 1 month prior for patients E, and F. Flows were quantified by cardiac-gated CMR, and thus they give in the best case cycle-averaged time-varying information. For patients $\mathrm{A}$ and $\mathrm{D}$, flows were measured in the branch pulmonary arteries; and for patients B, C, E and F, in the pulmonary veins, for which only time-averages are equal to the corresponding arterial flows. The total pulmonary flow (inflow) was obtained by adding the left and right sided pulmonary flows, and was imposed at the inlet surface (shunt). The mean values are reported in Table 1. Catheterizations were performed in the common atrium and on the venous side, by a pulmonary venous wedge pressure as a surrogate of the pulmonary artery pressure. Pressure differences between left and right sides were either measured or considered negligible $(0-1 \mathrm{mmHg})$, except for patient $\mathrm{D}$. The values defined as target pulmonary arterial pressures are reported in Table 1.

The main challenge of this study is to determine outflow boundary conditions for each patientspecific model that are consistent with the clinically measured data: 1) time-average flow distribution between left and right sides (flow split) denoted by fs and 2) pulmonary arterial pressure denoted by $P_{t}$, which is the target pressure to match, and which location and definition vary for each patient.

\subsection{Numerical methods}

The pre-operative anatomy of each patient was reconstructed from three-dimensional contrast enhanced CMR data using commercial software (Mimics, Materialise, NV, Leuven, Belgium). The vascular region of interest (the distal shunt and the pulmonary arteries extending to their first branches) was selected to include enough branches to avoid numerical instabilities or to largely include the future virtual stage 2 operative anastomosis [7,9], without going beyond the branches for which the image resolution would affect the simulation accuracy. A three-dimensional surface was thus obtained, following operations of segmentation and region-growing described in details in $[2,20]$.

For each of these 3D models, finite element meshes were generated with the commercial software MeshSim (Simmetrix Inc., Clifton Park, MY). Resulting meshes were anisotropically adapted to the flow in several steps $[12,19]$. In order to understand hemodynamics in these single ventricle geometries and reflect the effect of the downstream vascular trees, rigid-wall Navier-Stokes equations were solved in three-dimensional models, coupled to zero-dimensional models (Figure 1). This multidomain approach and its monolithic numerical implementation are described in details in $[24,25]$. All simulations were performed with a custom version of finite element Navier-Stokes solver, SimVascular (http://simtk.org), assuming blood as an incompressible Newtonian fluid with a density of $1060 \mathrm{~kg} \mathrm{~m}^{-3}$ and a dynamic viscosity of $0.004 \mathrm{~Pa} \mathrm{~s}$. 


\subsection{Tuning of reduced parameters}

The automatic tuning of outflow boundary condition was introduced in [23] for patient-specific simulations of the stage 2 (Glenn) procedure. This method was developed to obtain 3-element Windkessel parameters containing proximal resistance $\left(R_{p}\right)$ to describe viscous effects into large arteries; capacitance $(C)$ representing the ability of large arteries to store and return blood flow during a cardiac cycle; and distal resistance $\left(R_{d}\right)$ representing viscous effects into small arteries. A first part consisted of iteratively tuning a total resistance at each outlet by coupling $3 \mathrm{D}$ Navier-Stokes equations to a resistance with a fixed point method until recovering: 1) the measured transpulmonary gradient between the superior vena cava and the left atrium, and 2) the pulmonary flow split. Here this method is extended in order to take into account the different locations of measured pulmonary arterial pressure: instead of only handling situations where the measured pressure coincides with the pressure at one boundary of the 3D domain (the inlet), the former can be more general as will be explained below. This changes the way the outlet parameters are updated at each tuning iteration, and possibly the algorithm convergence. The framework presented below is the same for steady and pulsatile simulations.

The target flow split (fs) is computed from the time-average right pulmonary $\left(Q_{R}\right)$ and left pulmonary $\left(Q_{L}\right)$ CMR flows as follow:

$$
\mathrm{fs}=\frac{Q_{\mathrm{R}}}{Q_{\mathrm{L}}+Q_{\mathrm{R}}}
$$

The mean flow rate $\left(Q_{i}\right)$ that is the target to reach through an outlet $i$ is assumed to be proportional to the surface area of this outlet $\left(S_{i}\right)$ :

$$
Q_{i} \propto S_{i}
$$

A power law could also be used if relevant [23]. A target average value for the flow rate at outlet $i$ is thus as a function of the inlet flow rate $\left(\mathrm{Q}_{\mathrm{in}}\right)$ coming from clinical measurements:

$$
Q_{i}=Q_{\mathrm{in}}\left(\delta_{i \mathrm{r}} \mathrm{fs}+\left(1-\delta_{i \mathrm{r}}\right)(1-\mathrm{fs})\right) \frac{S_{i}}{\sum_{j} S_{j} \delta_{i j}}
$$

where $\delta_{i \mathrm{r}}$ is the kronecker symbol equal to 1 if outlet $i$ is on the right side and 0 if not, and $\delta_{i j}$ is the kronecker symbol equal to 1 if outlet $j$ is on the same side as outlet $i$ and 0 if not.

A first value of $R_{i}$ at each outlet is estimated to initialize the algorithm. This serves as the boundary condition for a first 3D Navier-Stokes simulation that is run during enough time steps in order to reach stable state results, typically a few 100 time steps for steady simulations and four cardiac cycles for pulsatile simulations. Note here that even in case of steady inlet flow (so called "steady simulations"), the resulting 3D flow is unsteady due to its complex interaction with the patientspecific geometry. Therefore, we use a transient formulation even for steady boundary conditions.

By post-processing the results over the last stable period, time-averaged (and mean in space) pressure $\left(P_{i}^{3 \mathrm{D}}\right)$ and flow rate $\left(Q_{i}^{3 \mathrm{D}}\right)$ are computed at each outlet. A flow split $\left(\mathrm{fs}^{3 \mathrm{D}}\right.$, based on the sum of the flows $Q_{i}^{3 \mathrm{D}}$ on each side), and a controlled pressure $\left(P_{c}^{3 \mathrm{D}}\right)$ that varies according to the measurement location and clinician input, are then calculated. Note that the exact pressure measurement location was unknown, hence this controlled pressure could not be related to the output pressure at a specific location in the 3D domain. Rather, if catheterization was performed on the left pulmonary side, $P_{c}^{3 \mathrm{D}}$ is the average pressure over all the outlets of the left side 
$\left(P_{i}^{3 \mathrm{D}}, i \in[0, N]\right.$ for $N$ the number of left pulmonary outlets). Sometimes, there were several measurements done on one or both sides; $P_{c}^{3 \mathrm{D}}$ is then defined with the clinical expert as being the maximum or the minimum value over all the corresponding branches of number $N$, e.g.

$$
P_{c}^{3 \mathrm{D}}=\min _{i} P_{i}^{3 \mathrm{D}}, i \in[0, N]
$$

These different options define the measurement for tuning and control method reported in Table 1. The results are then compared to the target pressure and flow values,

$$
\begin{aligned}
& \varepsilon_{P}=\frac{\left|P_{t}-P_{c}^{3 \mathrm{D}}\right|}{P_{t}} \\
& \varepsilon_{Q}=\left(\frac{1}{N} \sum_{i=1}^{N}\left(\frac{Q_{i}^{3 \mathrm{D}}-Q_{i}}{Q_{i}}-\frac{1}{N} \sum_{i=1}^{N} \frac{Q_{i}^{3 \mathrm{D}}-Q_{i}}{Q_{i}}\right)^{2}\right)^{\frac{1}{2}}
\end{aligned}
$$

where $\mathrm{N}$ is the total number of outlets.

If convergence is not reached within a certain tolerance, the resistance at each outlet is updated as follows:

$$
R_{i}=\frac{P_{t}-\left(P_{c}^{3 \mathrm{D}}-P_{i}^{3 \mathrm{D}}\right)}{Q_{i}}
$$

Another $3 \mathrm{D}$ simulation is run coupled to the new set of resistances $R_{i}$, where $i \in[0, N]$ for $N$ outlets. This process is repeated until convergence.

When pulsatile tuning simulations are performed, which can be important to better capture nonlinear effects across the 3D domain, resistances are also applied as boundary conditions, while pulsatile flow is imposed at the inlet. The controlled variables $\left(P_{c}^{3 \mathrm{D}}, Q_{i}^{3 \mathrm{D}}\right)$ are then the timeaveraged values over the last cardiac cycle. The steady tuning simulations were performed for all the six patients, whereas the pulsatile ones only for patients A and E due to time-varying arterial inflow issues. An additional step could be naturally included between the update of equation 6 and the next 3D iterative run, to update and impose more complex downstream reduced models, such as the one presented in the next section. This was not done here because the time-varying target pressure measurements were not available. Hence for each outlet, the 5-parameter reduced model was derived from the total resistance, with a combination of modeling and literature data. This is the object of the next section.

\subsection{Reduced model parameters for pulsatile simulation}

From the pulmonary arteries to the left atrium the vascular tree is carrying blood though the lung where gas exchanges occur. We divide this vasculature in three parts: the arteries downstream of the 3D geometry, the smaller arteries and capillaries, and the veins; representing the viscous effects and the ability of the larger vessels (both arteries and veins) to store and return blood during the cardiac cycle. The resulting parameters described on Figure 1 need thus to be determined. Assuming that pressures return periodically to the same values in the 5-parameter reduced model, the proximal arterial resistance $R_{p}^{\mathrm{ART}}$, the distal arterial resistance $R_{d}^{\mathrm{ART}}$ and the venous resistance $R_{d}^{\mathrm{VEIN}}$ can be summed to recover the total resistance at outlet $i$,

$$
R_{i}=R_{p}^{\mathrm{ART}}+R_{d}^{\mathrm{ART}}+R_{d}^{\mathrm{VEIN}}
$$




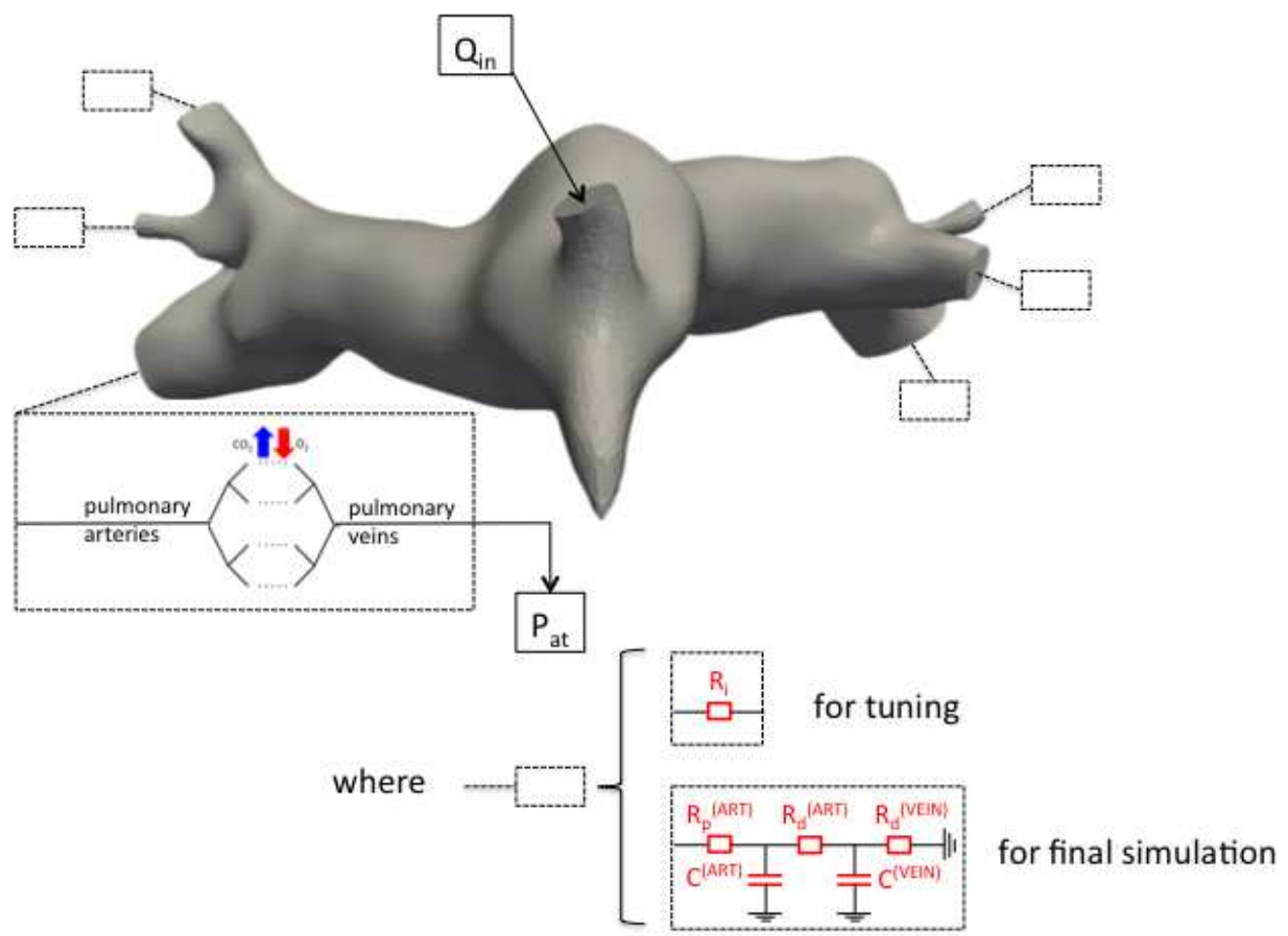

Fig. 1 Multiscale set-up, with the 3D domain, its inlet face on which the inflow $Q_{i n}$ is prescribed, and the distal pulmonary circulation for each outlet that all merge in the single atrium defined by its pressure $\mathrm{P}_{a t}$. At each outlet (dashed rectangle), the pulmonary arteries, capillaries and veins are represented either by a resistance for tuning, or by a more complete 5-parameter reduced model for final representation.

Next, empirical laws determine the distribution of the equivalent resistance and capacitance over the arterial or venous sides $[6,13,17]$. Thus for each outlet, $R_{d}^{\mathrm{VEIN}}=0.52 R_{i}$. The total pulmonary artery resistance $\left(R_{p}^{\mathrm{ART}}+R_{d}^{\mathrm{ART}}=0.48 R_{i}\right)$ leads to the localization of the branch within a morphometric pulmonary artery tree, and to the definition of the corresponding arterial sub-tree. A Windkessel model consisting of two resistances $\left(R_{p}^{\mathrm{ART}}, R_{d}^{\mathrm{ART}}\right)$ and one capacitance $\left(C^{\mathrm{ART}}\right)$ is generated from the Womersley-based impedance of this sub-tree [21,23]. Hence the proximal to distal pulmonary artery resistance ratio is different for each outlet. The capacitance in the venous tree is 5.7 bigger than in the arterial side. Combining all these relations determines a unique set of reduced parameters for each outlet i based on its total resistance.

In a next step, the three dimensional Navier-Stokes equations can be coupled to these reduced models at the outlets for more complete pulsatile simulations. A preoperative overview of the hemodynamics in the pulmonary arteries of each patient is thus generated in order to investigate a palliative surgery $[7,9]$. 


\section{Results}

The Results section presents the demonstration of this framework on six stage 1 single ventricle patients, defining their specific simulations parameters and reporting their 3D pressure, flow and wall shear stress.

\subsection{Outputs of simulation set up for each patient}

Figure 2 shows the reconstructed 3D geometries of patients A, B, C, D, E, and F.

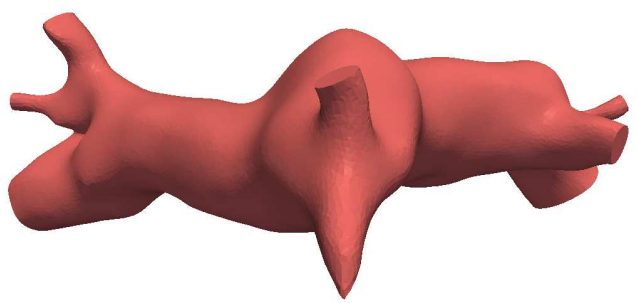

A
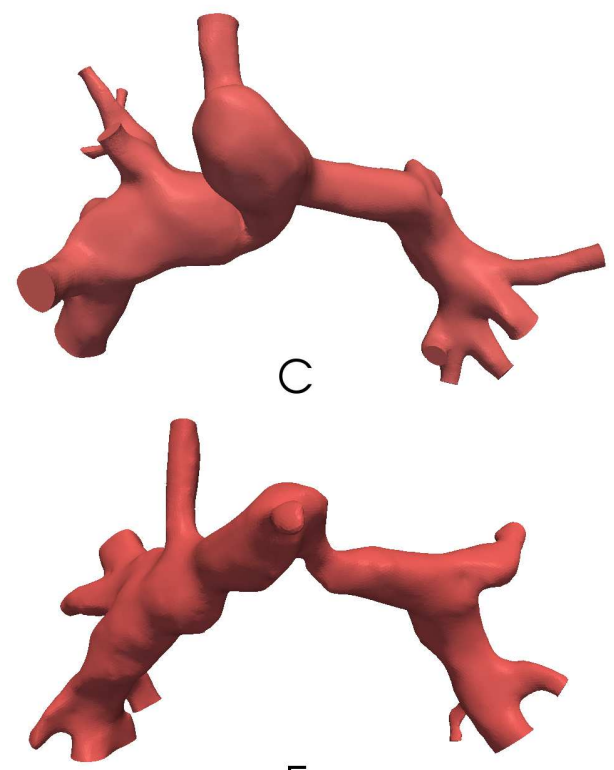

E

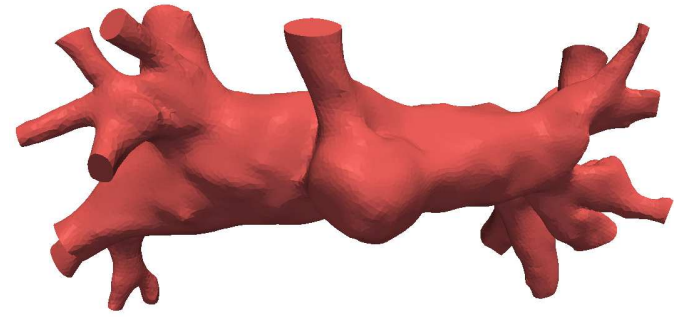

B

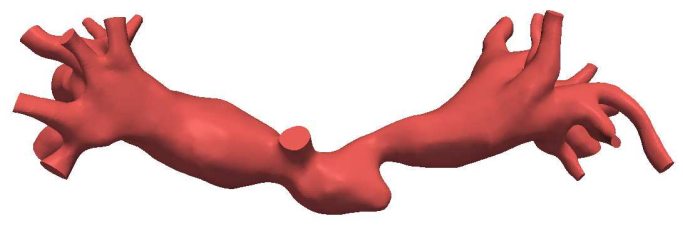

D

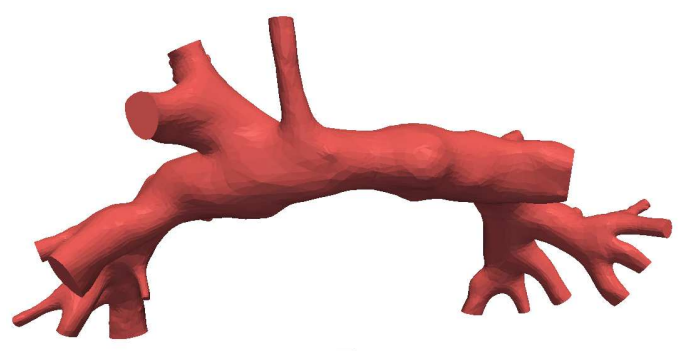

F

Fig. 2 3D geometrical model of patients A, B, C, D, E, F.

All 3D geometrical models included an inlet which is the distal shunt and a number of pulmonary branch outlets as described in the methods. The inlet surface areas of patients A, B, C, D, E, and $\mathrm{F}$ are respectively $3.3910^{-2}, 1.2710^{-1}, 1.8310^{-1}, 6.2110^{-2}, 6.2910^{-2}$ and $5.9310^{-2} \mathrm{~cm}^{2}$. The 3D meshes of patients A, B, C, D, E and F contain respectively $715 \mathrm{~K}, 970 \mathrm{~K}, 1050 \mathrm{~K}, 1500 \mathrm{~K}$, 
$710 \mathrm{~K}$ and $1540 \mathrm{~K}$ tetrahedra. Recall that the clinical data are the flow split between the right and left lungs and a pulmonary pressure. For patients A, B, C, D, E, and F, we should recover a flow split equal to $0.64,0.46,0.55,0.46,0.67$ and 0.52 respectively, and a pulmonary arterial pressure equal to $12.8,12.7,13.5,12,14$ and $11 \mathrm{mmHg}$ respectively, depending on the location where the clinical measurements were performed. Table 1 reports the pressure measurement location chosen for the tuning and the controlled value (average, minimum or maximum over branches of the measurement location) that was set by the clinicians. The pressure difference between the right and left pulmonary artery sides was negligible, except for patient D where it was $3 \mathrm{mmHg}$. Table 1 also reports the tuning results. Steady tuning was run for every patient: the results are very close to the targeted clinical data. For the first patient, pulsatile tuning was also performed for patient A and led to results of 0.64 for the flow split, $12.4 \mathrm{mmHg}$ for the pulmonary pressure and $0.4 \mathrm{mmHg}$ for the pressure difference, all very close to both steady tuning and targeted clinical values. For patient E, who was the only case with significant pressure difference between right and left lungs, results are $0.67,17 \mathrm{mmHg}$ and $3 \mathrm{mmHg}$ respectively, also very close to both steady tuning and targeted clinical values. As a consequence, the next paragraphs only report the $3 \mathrm{D}$ results of the steady tuning.

\begin{tabular}{|c|c|c|c|c|c|c|}
\hline Patient & $\mathrm{A}$ & $\mathrm{B}$ & $\mathrm{C}$ & $\mathrm{D}$ & $\mathrm{E}$ & $\mathrm{F}$ \\
\hline Age $(\mathrm{months})$ & 6 & 4 & 5 & 5 & 3 & 4 \\
\hline BSA $\left(\mathrm{m}^{2}\right)$ & 0.34 & 0.28 & 0.34 & 0.30 & 0.26 & 0.27 \\
\hline Inflow $\left(\mathrm{cm}^{3} / \mathrm{s}\right)$ & 7.5 & 12 & 20 & 9.7 & 12 & 16.6 \\
\hline Re $_{\max }$ & 2300 & 1750 & 2900 & 2500 & 3000 & 4150 \\
\hline fs & 0.64 & 0.46 & 0.55 & 0.46 & 0.67 & 0.52 \\
\hline PA pressure $(\mathrm{mmHg})$ & 12.8 & 12.7 & 13.5 & 12 & 17 & 11 \\
\hline$\Delta \mathrm{P}_{\mathrm{L} / \mathrm{R}}(\mathrm{mmHg})$ & negl. & negl. & negl. & negl. & 3 & negl. \\
\hline Meas. location for tuning & both & LPA & LPA & both & RPA & both \\
\hline Control method & average & average & average & average & min & max \\
\hline fs & $0.64(0.64)$ & 0.46 & 0.55 & 0.46 & $0.66(0.67)$ & 0.52 \\
\hline$P_{c}^{3 D}(\mathrm{mmHg})$ & $12.3(12.4)$ & 12.7 & 13.4 & 12.0 & $17.0(17)$ & 11.0 \\
\hline$\Delta \mathrm{P}_{\mathrm{L} / \mathrm{R}}^{3 \mathrm{D}}(\mathrm{mmHg})$ & $0.4(0.4)$ & 0.7 & 0.5 & 6.0 & $3.0(3)$ & 0.2 \\
\hline
\end{tabular}

Table 1 Clinical measurements, numerical method and results. For each patient, clinical data (age, BSA, average pulmonary inflow, deduced Reynolds number $R e_{\max }$, flow split fs, target pulmonary artery pressure value, negligible or not left/right pulmonary pressure difference), tuning set-up (left or right pulmonary side, and control method to define $P_{c}^{3 \mathrm{D}}$ ), and numerical results in red (obtained flow split fs ${ }^{3 \mathrm{D}}$, controlled pressure $P_{c}^{3 \mathrm{D}}$ and pressure difference between left and right sides $\Delta \mathrm{P}_{\mathrm{L} / \mathrm{R}}^{3 \mathrm{D}}$ ). Numbers are for the steady tuning and in parenthesis for the pulsatile tuning.

\subsection{Pressure distribution}

We present in Figure 3 the pressure map for each patient at the end of the tuning. We observe a high peak of pressure on the wall. The maximal pressure for patients A, B, C, D, E and F are respectively 52, 26, 25, 45, 62 and $74 \mathrm{mmHg}$. The patients $\mathrm{A}, \mathrm{B}$ and $\mathrm{F}$ have homogeneous pressure in both pulmonary arteries respectively, approximately 12, 13 and $11 \mathrm{mmHg}$. Moreover, patients $\mathrm{D}$ and $\mathrm{E}$ have a left pulmonary artery (LPA) stenosis which involves a significant pressure loss of approximately $3 \mathrm{mmHg}$ across the stenosis, while homogeneous pressure is found in the right pulmonary artery (RPA). A last remark concerns patient $\mathrm{C}$ who has a kink in the RPA close to the shunt. The kink does not generate a significant pressure loss and pressure is homogeneous in both PAs, around $13.5 \mathrm{mmHg}$. 


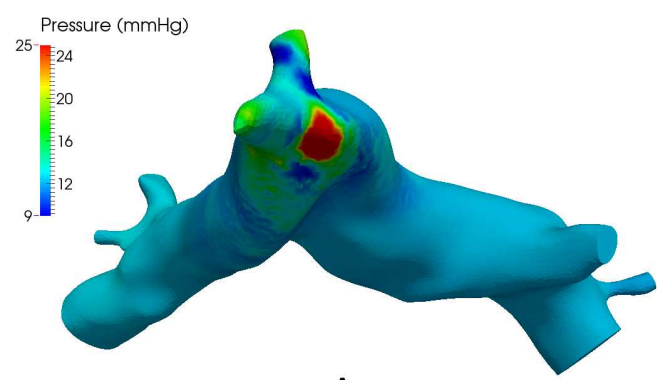

A
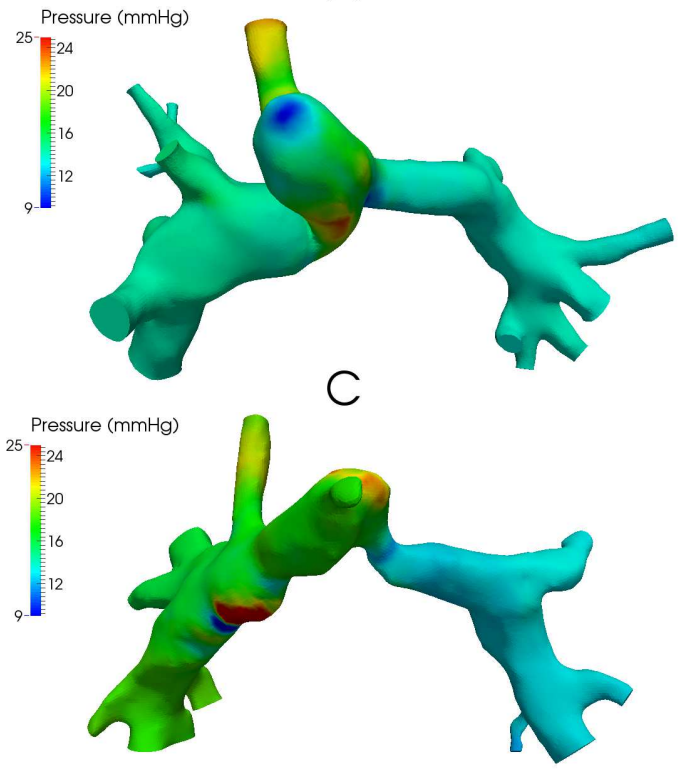

$\mathrm{E}$

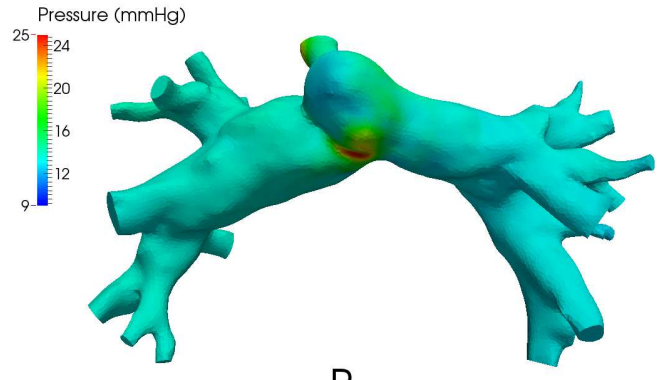

B

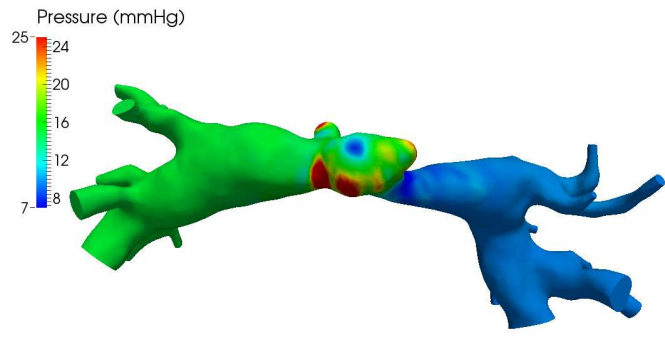

D

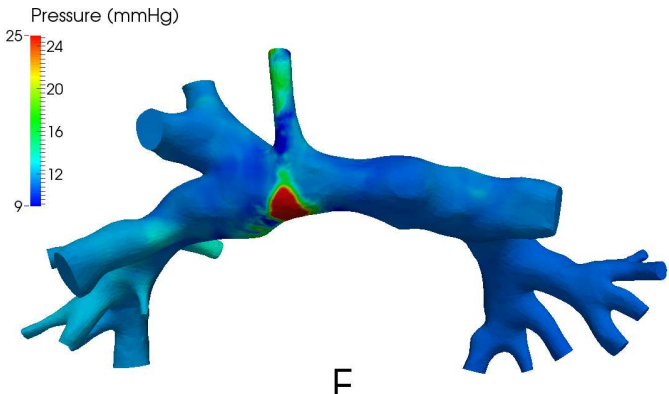

Fig. 3 Pressure maps for patients A-F. Maximum pressure is equal to $25 \mathrm{mmHg}$ on the color scale, even if the real maximal pressure is larger. $1 \mathrm{mmHg}=133.3 \mathrm{~Pa}$

\subsection{Velocity patterns}

In Figure 4 blood flow patterns for each patient are presented at the end of the tuning. A very complex flow can be observed in the 3D geometrical domain, especially close to the shunt where blood flow is the highest. Highest velocity is obtained in the center of the shunt and is respectively equal to $470,250,265,319,377$ and $570 \mathrm{~cm} / \mathrm{s}$. For patients $\mathrm{A}, \mathrm{B}$, and $\mathrm{F}$ the blood is swirling in the pulmonary arteries close to the shunt and goes towards the outlet smoothly with less complexity. This mixing flow behavior occurs into the main pulmonary artery stump for patients $\mathrm{B}, \mathrm{C}$, and $\mathrm{D}$ where the anastomosis of the ligated main pulmonary artery is prominent. For patient E, swirling reflects the tortuous geometry on the left side, where there are a constricted anastomosis of the ligated main pulmonary artery, followed by a stenosis. 


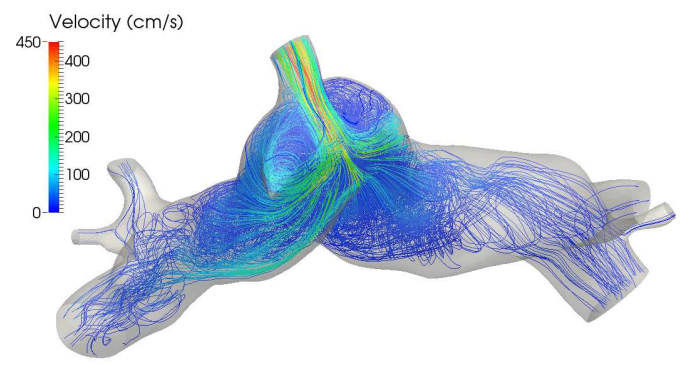

A

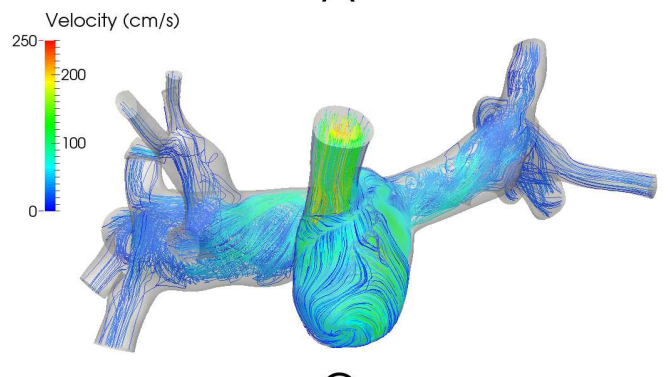

C

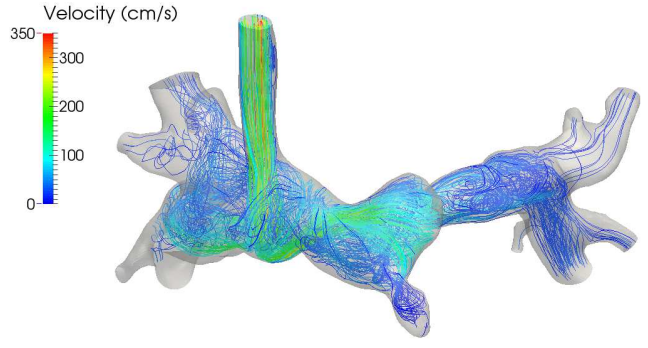

$\mathrm{E}$

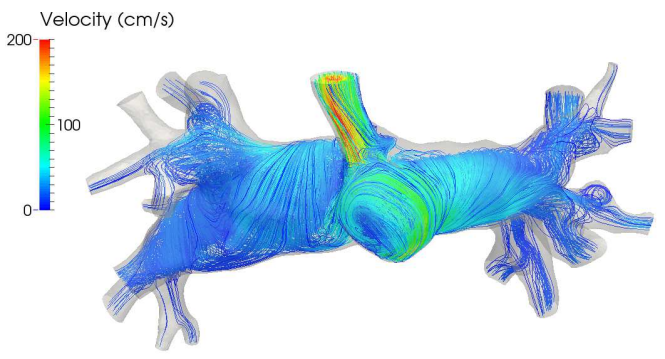

B

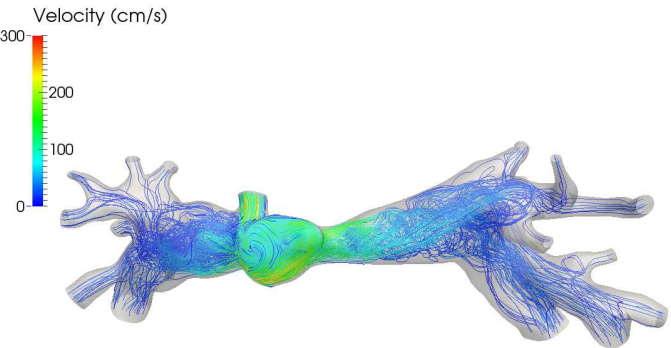

$\mathrm{D}$

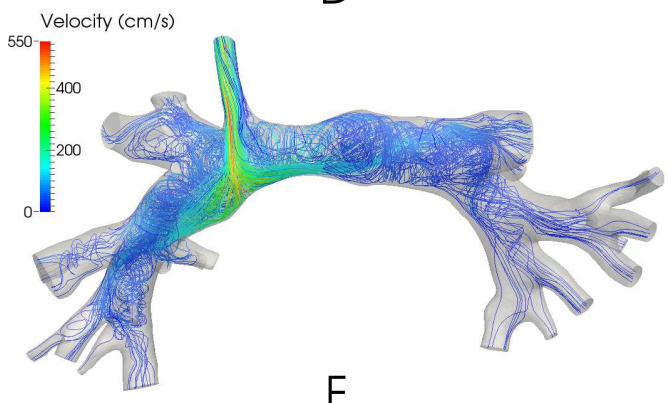

Fig. 4 Streamlines of patients A-F colored by velocity magnitude.

\subsection{Wall shear stress maps}

In Figure 5 the wall shear stress surface maps are represented for each patient. For all patients the maximal wall shear stress is reached either in the shunt, or where there is a peak of pressure, respectively equal to $73.8,55.3,52.9,66.3,67.1$, and $69.7 \mathrm{~Pa}$.

Moreover, for patients $\mathrm{D}$ and $\mathrm{E}$, the stenosis involves a large wall shear stress respectively equal to 54.3 and $61.4 \mathrm{~Pa}$. Regarding patient A, where the main pulmonary artery was ligated the mean wall shear stress is $57.8 \mathrm{~Pa}$, which is high compared to the rest of the domain. In contrast, in the corresponding location of patient $\mathrm{E}$, the mean wall shear stress is close to 0 .

Comparing patients $\mathrm{B}$ and $\mathrm{C}$, which have a similar shape, the obtained mean wall shear stress is respectively equal to 17 and $40 \mathrm{~Pa}$. 


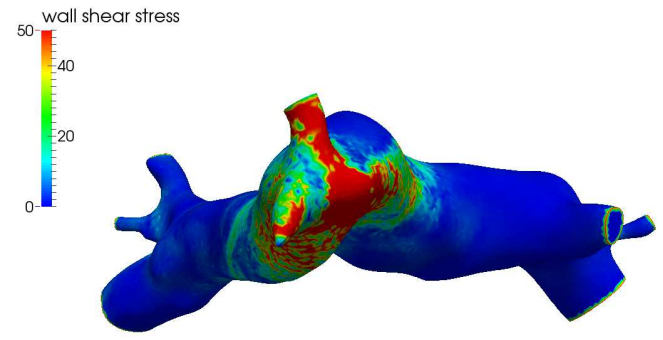

A

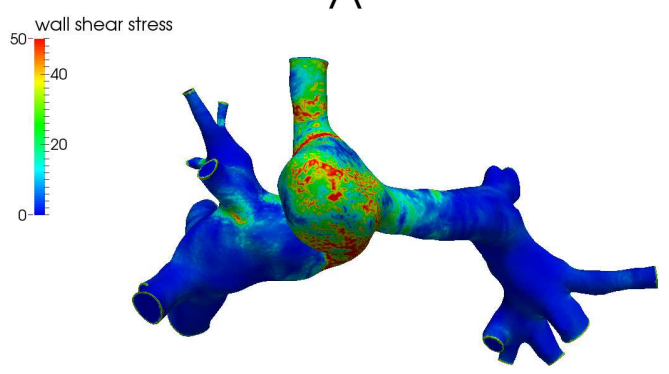

C

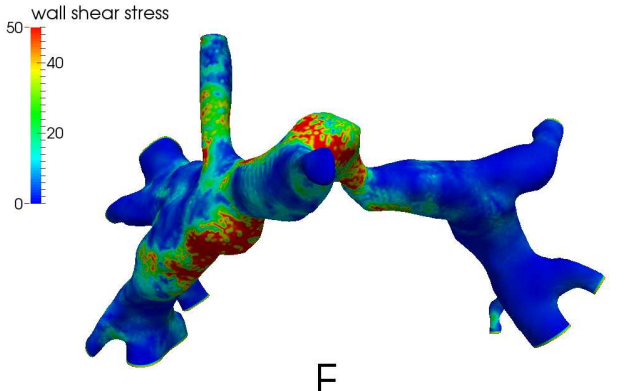

$\mathrm{E}$

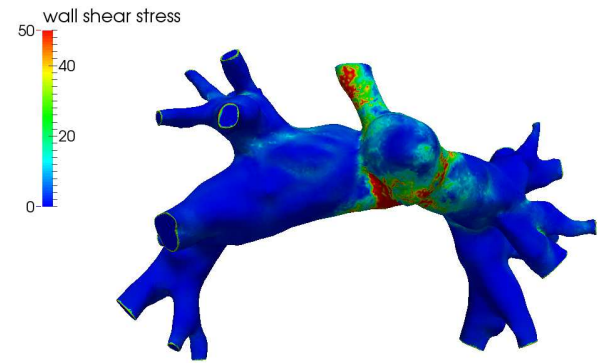

B

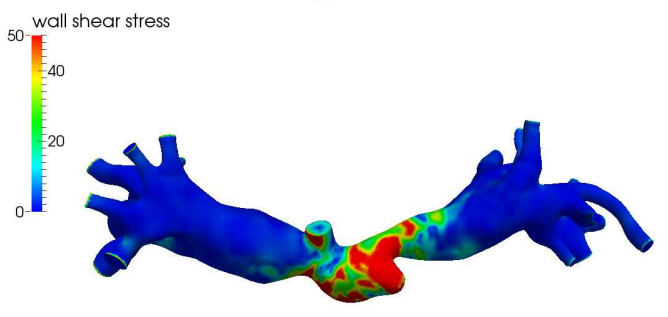

D

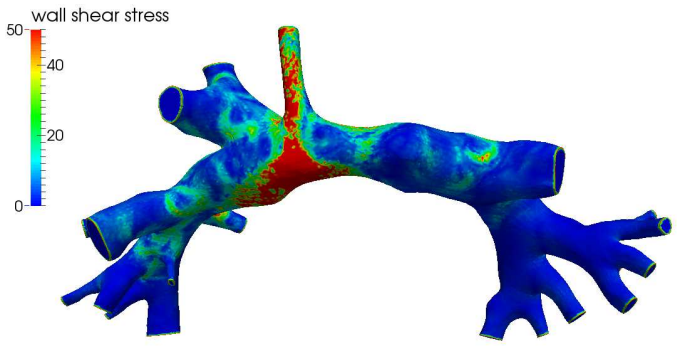

$\mathrm{F}$

Fig. 5 Wall shear stress map of patients A-F in Pa. Maximum color scale is fixed at $50 \mathrm{~Pa}$ but the real maximum value is larger.

\section{Discussion}

\subsection{Significance of the Results}

The proposed methodology to tune reduced model parameters accurately replicates clinical measurements 1, at least within acceptable clinical measurement tolerance. Moreover, we highlight the high complexity of the blood flow patterns in pulmonary arteries in the context of the stage 1 single ventricle procedure. Patients have a BSA between 0.26 and $0.34 \mathrm{~m}^{2}$. The variability of the pulmonary flow rate - between 7.5 and $20 \mathrm{~cm}^{3} / \mathrm{s}$ - leads to very high Reynolds numbers, between 1750 and 4150 when computed at the shunt inlet with the maximum velocity.

This leads to values of wall shear stress that are very high. They are in the same order of magnitude as in the aorta of a typical Norwood patient [18] (keeping in mind that the shunt is a direct connection from the aorta or brachial artery). In the Glenn circulation [23], a typical wall shear stress is more than 10 times lower. For patients B and C, the main pulmonary artery was 
ligated, forming a sphere bulging out of the anastomosis, which might foster recirculation and thus low wall shear stress. Yet, the computed wall shear stresses were quite different. The relative differences in wall shear stress between patients may have some bearing on the development of the PAs, however, the stage 1 circulation is only present for a few months before second stage palliative surgery is required. Therefore, these effects will be of a fairly short duration.

The importance of the severe stenosis for the pressure drop and the swirling behavior of the blood flow was also shown. This complexity is induced by the high Reynolds number is in the shunt. For these stenosis cases and patient $\mathrm{C}$ with its kinks, computational fluid dynamics is a a complement to clinical measurements. One potential clinical application for this technique is to aid clinicians understanding of the significance of anatomical abnormalities in these complex patients. For example, patient $\mathrm{C}$ appears to have a significant geometric 'kink' or restriction in their RPA just distal to the shunt. Only a distal LPA pressure measurement was obtained clinically, so the hemodynamic effect of this lesion was unknown. However, simulation results clearly indicate that the pressure difference between the shunt anastomosis and the PAs are equal on the left and right sides. This was of interest to the clinicians, as the consensus was that judging by the anatomy alone, they would expect a pressure loss across the RPA. Indeed, at stage 2 surgery, patients $\mathrm{A}, \mathrm{C}, \mathrm{D}$, and $\mathrm{E}$ all underwent patch augmentation of their central PAs or proximal LPAs judged on the appearance of the geometry alone. This work suggests that in some cases the clinician's perception of what constitutes a geometric abnormality may actually not result in a hemodynamically significant pressure difference, as in case C. Although, the tortuous course of the PA appears to constitute a stenosis, it is partly an illusion due to the distal PA having a relatively large cross-sectional area.

For patient $\mathrm{D}$, a first attempt was to match the measured flow split of 0.46 and a pressure at both LPA and RPA side equal to $12 \mathrm{mmHg}$. Unfortunately, targeting the flow split to be 0.46 induced a pressure difference between left and right sides around $6 \mathrm{mmHg}$ which was not acceptable from a clinical point of view. Discussions with clinicians led us to disregard the clinically measured flow split. By imposing the same pressure on average on each side, a flow split of 0.54 (more flow to the right side) was then found, which was clinically acceptable. The differences in flow split would correspond to an error of $0.78 \mathrm{ml} / \mathrm{s}$ (i.e. $8 \%$ ) in estimating flow with CMR. This example highlights the impact of uncertainty of measurements on the numerical simulations. It could be interesting to investigate more precisely the effect of uncertainties of pressure and flow split measurements on the pressure loss through the stenosis. This patient-specific example was thus challenging and required a close collaboration with clinicians. On the other hand, performing simulations underlined the incoherence between the original clinical measurements, and helped to assess together with the clinical experts which data made more sense to trust.

\subsection{Methodology Discussion}

The tuning methodology introduced in this work consists of coupling Navier-Stokes equations to reduced models (here resistances) at the outlets and running steady or pulsatile simulations. The a priori interest of running pulsatile simulation is the integration of all non linearities due to the $3 \mathrm{D}$ geometrical model and obtain a more accurate set of reduced model parameters. However, to achieve periodic stability, pulsatile simulations need to be run over 4 cardiac cycles (around 2000 time steps) and the iterative process is converging in 5 iterations, thus around 10000 time steps. In comparison, 100 time steps for a steady stimulation were enough and the iterative process converges in 4 iterations, thus around 400 time steps were necessary in total. Furthermore, pulsatile 
tuning for patients $\mathrm{A}$ and $\mathrm{E}$ were performed and both matched targeted clinical values and were very close to the steady tuning results. Moreover, the reduced model parameters were very close to those obtained by steady tuning. Indeed, for patients $\mathrm{A}$ and $\mathrm{E}$ the difference between steady and pulsatile tuning are respectively $6.37 \%$ and $7.82 \%$ in resistances, computed by the formula (5). Note that the same framework could be used to tune total capacitance values for example or even more parameters, if pulsatile pressure measurements were available in synchrony with the flow measurements [15].

For sake of brevity, the 5 parameters of each branch for the six patients considered in this study are not presented in the results section. Such reduced models of the pulmonary circulation for each outlet have been incorporated into preoperative closed-loop simulations representative of the patient state before surgery. They have been then used to investigate different surgical options as in $[7,9]$, keeping all these outlet blocks the same while changing the $3 \mathrm{D}$ part and its connecting inflow. Deriving this 5-parameter model of the arterial and venous pulmonary circulation has lead to more successful matching clinical time-varying tracings in the closed-loop simulations than the previous 3-component Windkessel model that neglected in particular the venous compliance.

A first source of uncertainty comes from the geometrical reconstruction for these young babies. The typical pixel size from 3D CMR angiography is $1 * 1 \mathrm{~mm}^{2}$. In addition 3D CMR angiography may suffer from signal loss in areas of turbulent or complex flow. Thus including or excluding a pixel in a small area region, such as a shunt stenosis, may lead to errors in the 3D geometry. This kind of uncertainty should be addressed in future work. Where available, 2D CMR black blood imaging sequences were used to check diameters at specific locations since spatial resolution is higher in this sequence. As a final check for each patient, a visual comparison was made between the $3 \mathrm{D}$ reconstruction and the $2 \mathrm{D}$ cardiac catheter angiography sequences. Patient $\mathrm{D}$ had the appearance of luminal narrowing with an obvious distal stenosis on catheter angiographic sequences. The $2 \mathrm{D}$ measurements were used to adjust the $3 \mathrm{D}$ reconstruction to give an accurate representation of the geometry in this region.

Branching bifurcations were included evenly on both sides, coherently with image resolution. Although a large number of branches increases computational time, cutting the branches before the first bifurcations on each side often leads to numerical instabilities, as was tested for patient A. Stabilizing the numerical scheme is then warranted [1], [11]. Note that sometimes, as was the case for patient $\mathrm{F}$, the robust convective stabilization was not enough to avoid numerical divergence in the short model. Besides, as cutting branches or stabilization can change flow features close to the boundary, it is necessary to include enough branches to not affect the flow close to the current anastomosis or the one of the next stage virtual surgery.

Furthermore, in this work walls of the 3D geometrical models were assumed rigid because data for the heterogeneous assessment of the wall parameters were not known. Moreover, the shunt is a rigid graft and the anastomosis is also quite rigid because of sutures. If time-varying geometry data were available, the elasticity parameters could also be inferred [4]. However, it has been shown that FSI has little effect at rest at least at later stage of the palliation [3].

Regarding the inlet boundary condition, since the velocity profile was not available, a parabolic profile was imposed at the inlet, which is commonly used for blood flow in arteries. We verified on one case that changing the profile to a flat velocity profile did not significantly change the 
pressure or the velocity fields.

The clinical hemodynamics measurements have uncertainties and it would be interesting to take it into account in a future work [23]. Indeed it would be useful to investigate their effect on the pressure loss through stenosis and on the set of reduced model parameters.

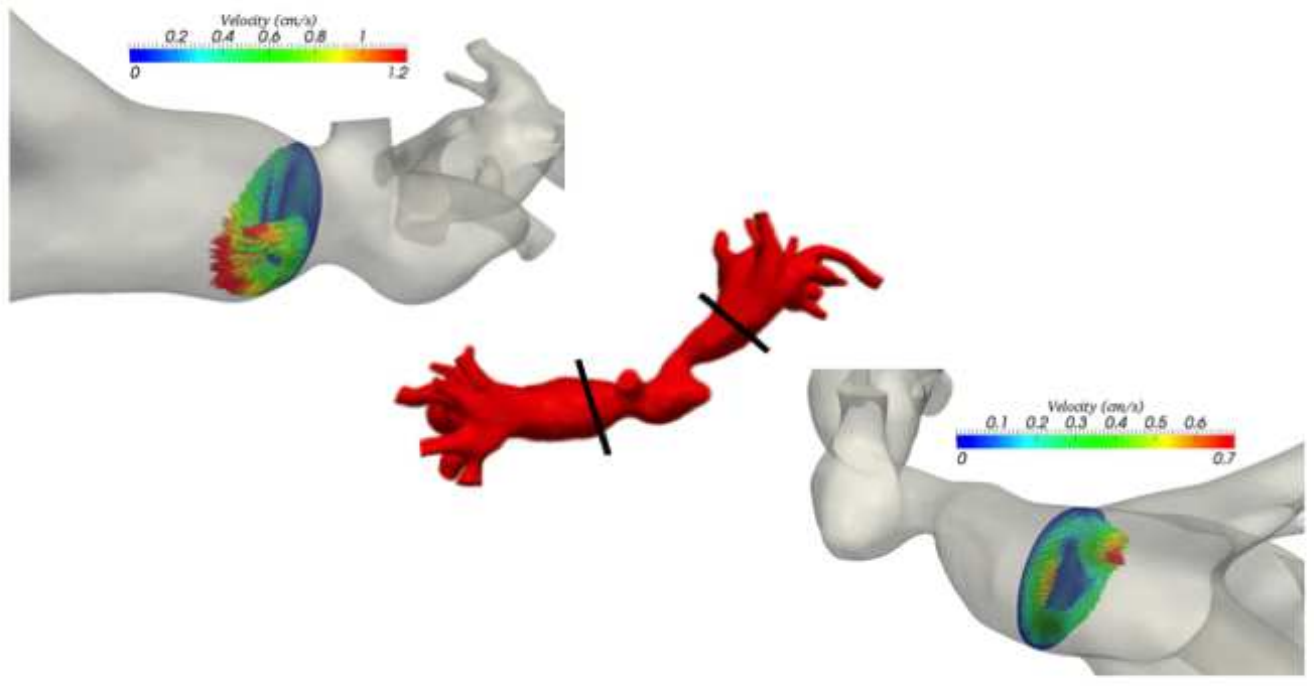

Fig. 6 Velocity vectors (coloured by their magnitudes) from the 3D simulation shown at the locations where the CMR flow measurements were performed.

A last comment regarding uncertainties of clinical measurements: in Figure 6 simulated velocity vectors for patient $\mathrm{D}$ are shown at the same locations as where flow measurements were done in CMR. We highlight the complexity of the blood flow patterns at these locations, which might explain the difficulty to get accurate clinical time-varying velocity measurements by CMR at these locations. The PAs are relatively small, and are receiving flow from the aorta, or one of its branches, at high pressure. CMR flow measurements are typically less reliable in areas of complex flow because unpredictable phase shifts occur. This often leads to signal loss (voiding) and inaccurate flow measurements [14]. Typically, the PA flow measurements in this location would be underestimated in stage 1 patients. The pulmonary veins may represent a more stable location to measure the pulmonary flow split, however, the physiology of these patients sometimes leads to the pulmonary veins receiving additional blood flow external to the pulmonary arteries. 
It is only through the iterative process of comparing simulation results to clinical measurements that suitable strategies for managing measurement uncertainty are reached.

\section{Conclusion}

In this work we develop a method to iteratively tune parameters of reduced models of 3D blood flow simulations in order to reflect the effect of the downstream vasculature, taking into account clinical measurements of stage 1 single ventricle patients before they underwent the next palliative operation. This method was effectively demonstrated on six patients. It can be useful in other multi-branched blood flow simulations for which pressure or flow measurements are taken at various locations, and chosen to be matched as a minimum, maximum, average or other combinations of values in a given region. Simulations in these stage 1 cases highlighted the complexity of the blood flow patterns into the shunt anastomosis and the pulmonary arteries, as well as the very specific pressure map. The wall shear stress was found to be very high in magnitude, but varying in space differently according to the patients anatomy, not necessarily leading to a low value in the ligated main pulmonary stump. Simulations also assessed the effects of anatomical kinks or stenoses, demonstrating the potential use of the technique: clinicians do not always know whether a lesion is hemodynamically significant. These types of simulations could be helpful when planning whether to intervene on a PA anatomical problem. It involves a lot more surgery to intervene on a PA stenosis so if clinicians better understood the difference between a significant stenosis and an anatomical anomaly that does not have an effect, it would save time and danger intervening when it was not necessary.

This methodology is moreover helpful for virtual surgical planning testing different surgical approaches $[7,9]$. It is also shown to be a tool for clinicians to assess coherence of clinical measurements : the iterative approach between comparing clinical data and the simulation results emerged as efficient to understand the limitations in clinical measurements and decide upon a strategy to replicate the clinical case that makes sense to clinicians. This process raises the issue of the impact of uncertainty quantification of the clinical measurements.

Finally, this first work on single ventricle shunt anastomoses at stage 1 could be used to compare hemodynamics of different stages of the Fontan palliation.

Acknowledgements This work was supported by the Leducq Foundation as part of the Transatlantic Network of Excellence for Cardiovascular Research "Multi-scale modeling of single ventricle hearts for clinical decision support", and by the Associated team Cardio INRIA grant.

Conflict of interest: None.

\section{References}

1. Arbia, G., Gerbeau, J.F., Hsia, T.Y., Vignon-Clementel, I.E.: A new approach for the outflow boundary conditions in three-dimensional hemodynamics. Prep.

2. Armillotta, A., Bonhoeffer, P., Dubini, G., Ferragina, S., Migliavacca, F., Sala, G., Schievano, S.: Use of rapid prototyping models in the planning of percutaneous pulmonary valved stent implantation. Proc. Inst. Mech. Eng. Part H J. Eng. Med. 221(4), 407-416 (2007). DOI 10.1243/09544119JEIM83. URL http://pih.sagepub.com/content/221/4/407.abstract

3. Bazilevs, Y., Hsu, M.C., Benson, D.J., Sankaran, S., Marsden, A.L.: Computational fluid-structure interaction: methods and application to a total cavopulmonary connection. Comput. Mech. 45(1), 77-89 (2009). DOI 10.1007/s00466-009-0419-y. URL http://dx.doi.org/10.1007/s00466-009-0419-y 
4. Bertoglio, C., Moireau, P., Gerbeau, J.F.: Sequential parameter estimation for fluid-structure problems: Application to hemodynamics. Int. j. numer. method. biomed. eng. 28(4), 434-455 (2012)

5. Bove, E.L., de Leval, M.R., Migliavacca, F., Guadagni, G., Dubini, G.: Computational fluid dynamics in the evaluation of hemodynamic performance of cavopulmonary connections after the Norwood procedure for hypoplastic left heart syndrome. J. Thorac. Cardiovasc. Surg. 126(4), 1040-1047 (2003). DOI 10.1016/S00225223(03)00698-6. URL http://linkinghub.elsevier.com/retrieve/pii/S0022522303006986

6. Brody, J.S., Stemmler, E.J., DuBois, A.B.: Longitudinal distribution of vascular resistance in the pulmonary arteries, capillaries, and veins. J. Clin. Invest. 47, 783-799 (1968). DOI 10.1172/JCI105773

7. Corsini, C., Baker, C., Kung, E., Schievano, S., Arbia, G., Baretta, A., Biglino, G., Migliavacca, F., Dubini, G., Pennati, G., Marsden, A.L., Vignon-Clementel, I., Taylor, a.M., Hsia, T.Y., Dorfman, A.: An integrated approach to patient-specific predictive modeling for single ventricle heart palliation. Comput. Methods Biomech. Biomed. Engin. (June), 37-41 (2013). DOI 10.1080/10255842.2012.758254. URL http://www.ncbi.nlm.nih.gov/pubmed/23343002

8. Ismail, M., Wall, W.A., Gee, M.W.: Adjoint-based inverse analysis of windkessel parameters for patient-specific vascular models. J. Comput. Phys. 244(0), 113-130 (2013). DOI http://dx.doi.org/10.1016/j.jcp.2012.10.028. URL http://www.sciencedirect.com/science/article/pii/S0021999112006298

9. Kung, E., Baretta, A., Baker, C., Arbia, G., Biglino, G., Corsini, C., Schievano, S., Vignon-Clementel, I.E., Dubini, G., Pennati, G., Others: Predictive modeling of the virtual Hemi-Fontan operation for second stage single ventricle palliation: two patient-specific cases. J. Biomech. 46(2), 423-429 (2013)

10. Migliavacca, F., Pennati, G., Dubini, G., Fumero, R., Pietrabissa, R., Urcelay, G., Bove, E.L., Hsia, T.Y., de Leval, M.R.: Modeling of the Norwood circulation: effects of shunt size, vascular resistances, and heart rate. Am. J. Physiol. - Hear. Circ. Physiol. 280(5), H2076--H2086 (2001). URL http://ajpheart.physiology.org/content/280/5/H2076

11. Moghadam, M.E., Bazilevs, Y., Hsia, T.Y., Vignon-Clementel, I.E., Marsden, A.L.: A comparison of outlet boundary treatments for prevention of backflow divergence with relevance to blood flow simulations. Comput. Mech. 48(3), 277-291 (2011)

12. Muller, J., Sahni, O., Li, X., Jansen, K.E., Shephard, M.S., Taylor, C.A.: Anisotropic adaptive finite element method for modelling blood flow. Comput. Methods Biomech. Biomed. Engin. 8(5), 295-305 (2005). DOI 10.1080/10255840500264742. URL http://www.tandfonline.com/doi/abs/10.1080/10255840500264742

13. O'Leary, C.E., Fiori, R., Hakim, T.S.: Perioperative distribution of pulmonary vascular resistance in patients undergoing coronary artery surgery. Anesth. Analg. 82, 958-963 (1996)

14. Oshinski, J.N., Ku, D.N., Pettigrew, R.I.: Turbulent Fluctuation Velocity: The Most Significant Determinant of Signal Loss in Stenotic Vessels. Magn. Reson. Med. 33(2), 193-199 (1995). DOI 10.1002/mrm.1910330208

15. Pant, S., Fabreges, B., Gerbeau, J.F., Vignon-Clementel, I.E.: A multiscale filtering-based parameter estimation method for patient-specific coarctation simulations in rest and exercise. Proc. 16th Int. Conf. Med. Image Comput. Comput. Assist. Interv. to appear (2013)

16. Pennati, G., Corsini, C., Cosentino, D., Hsia, T.Y., Luisi, V.S., Dubini, G., Migliavacca, F.: Boundary conditions of patient-specific fluid dynamics modelling of cavopulmonary connections: possible adaptation of pulmonary resistances results in a critical issue for a virtual surgical planning. Interface Focus 1(3), 297-307 (2011). DOI 10.1098/rsfs.2010.0021. URL http: //www.pubmedcentral.nih.gov/articlerender.fcgi?artid=3262435\&tool=pmcentrez\&rendertype=abstract

17. Presson, R.G., Audi, S.H., Hanger, C.C., Zenk, G.M., Sidner, R.A., Linehan, J.H., Wagner, W.W., Dawson, C.A.: Anatomic distribution of pulmonary vascular compliance. J. Appl. Physiol. 84, 303-310 (1998)

18. Qian, Y., Liu, J.L., Itatani, K., Miyaji, K., Umezu, M.: Computational Hemodynamic Analysis in Congenital Heart Disease: Simulation of the Norwood Procedure. Ann. Biomed. Eng. 38(7), 2302-2313 (2010). DOI 10.1007/s10439-010-9978-5. URL http://dx.doi.org/10.1007/s10439-010-9978-5

19. Sahni, O., Muller, J., Jansen, K., Shephard, M., Taylor, C.: Efficient anisotropic adaptive discretization of the cardiovascular system. Comput. Methods Appl. Mech. Eng. 195, 5634-5655 (2006). DOI $10.1016 /$ j.cma.2005.10.018

20. Schievano, S., Migliavacca, F., Coats, L., Khambadkone, S., Carminati, M., Wilson, N., Deanfield, J.E., Bonhoeffer, P., Taylor, A.M.: Percutaneous Pulmonary Valve Implantation Based on Rapid Prototyping of Right Ventricular Outflow Tract and Pulmonary Trunk from MR Data. Radiology 242(2), 490-497 (2007). DOI 10.1148/radiol.2422051994. URL http://pubs.rsna.org/doi/abs/10.1148/radiol.2422051994

21. Spilker, R.L., Feinstein, J.A., Parker, D.W., Reddy, M.V., Taylor, C.A.: Morphometry-Based Impedance Boundary Conditions for Patient-Specific Modeling of Blood Flow in Pulmonary Arteries. Ann. Biomed. Eng. 35(4), 546-559 (2007). DOI 10.1007/s10439-006-9240-3. URL http://dx.doi.org/10.1007/s10439-0069240-3

22. Spilker, R.L., Taylor, C.a.: Tuning multidomain hemodynamic simulations to match physiological measurements. Ann. Biomed. Eng. 38(8), 2635-48 (2010). DOI 10.1007/s10439-010-0011-9. URL http://www.ncbi.nlm.nih.gov/pubmed/20352338

23. Troianowski, G., Taylor, C.A., Feinstein, J.A., Vignon-Clementel, I.E., Others: Three-dimensional simulations in Glenn patients: clinically based boundary conditions, hemodynamic results and sensitivity to input data. Trans. ASME-K-Journal Biomech. Eng. 133(11), 111,006 (2011) 
24. Vignon-Clementel, I.E., Alberto Figueroa, C., Jansen, K.E., Taylor, C.a.: Outflow boundary conditions for three-dimensional finite element modeling of blood flow and pressure in arteries. Comput. Methods Appl. Mech. Eng. 195(29-32), 3776-3796 (2006). DOI 10.1016/j.cma.2005.04.014. URL http://linkinghub.elsevier.com/retrieve/pii/S0045782505002586

25. Vignon-Clementel, I.E., Figueroa, C.a., Jansen, K.E., Taylor, C.a.: Outflow boundary conditions for 3D simulations of non-periodic blood flow and pressure fields in deformable arteries. Comput. Methods Biomech. Biomed. Engin. 13(5), 625-40 (2010). DOI 10.1080/10255840903413565. URL http://www.ncbi.nlm.nih.gov/pubmed/20140798

26. Vignon-Clementel, I.E., Marsden, A.L., Feinstein, J.A.: A primer on computational simulation in congenital heart disease for the clinician. Prog. Pediatr. Cardiol. 30(1), 3-13 (2010)

27. de Zélicourt, D.A., Marsden, A., Fogel, M.A., Yoganathan, A.P.: Imaging and patient-specific simulations for the Fontan surgery: Current methodologies and clinical applications. Prog. Pediatr. Cardiol. 30(1), 31-44 (2010) 